\title{
Encounter-driven accretion in young stellar clusters - A connection to FUors?
}

\author{
S. Pfalzner \\ I. Physikalisches Institut, University of Cologne, Zülpicher Str. 77, 50937 Cologne, Germany
}

e-mail: pfalzner@ph1.uni-koeln.de

Received 29 August 2008 / Accepted 15 October 2008

ABSTRACT

\begin{abstract}
Context. The brightness of FUors increases by several magnitudes within one to several years. The currently favoured explanation for this brightness boost is that of dramatically rising accretion from the disc material around a young star. The mechanism leading to this accretion increase is a point of debate.

Aims. Choosing the Orion nebula cluster as representative, we simulate accretion bursts driven by encounters in dense stellar environments. We investigate whether properties like rise and decay times, event frequency, etc., speak for encounters as a possible cause for FUor phenomena.

Methods. We combine cluster simulations performed with the Nbody6++ code with particle simulations that describe the effect of a fly-by on the disc around a young star to determine the induced mass accretion.

Results. The induced accretion rates, the overall temporal accretion profile, the decay time, and possibly the binarity rate we obtain for encounter-induced accretion agree very well with observations of FUors. However, the rise time of one year observed in some FUors is difficult to achieve in our simulations unless the matter is stored somewhere close to the star and then released after a certain mass limit is transgressed. The severest argument against the FUors phenomenon being caused by encounters is that most FUors are found in environments of low stellar density. We extend the discussion to eccentric binaries and gravitationally unstable discs and find that both models have similar problems in achieving the necessary rise times.

Conclusions. We find no conclusive answer as to whether the observed FUors are triggered by encounters. However, it seems an intense accretion burst phase should exist - possibly an FU phase - early on in the development of dense clusters. We predict that in dense young clusters these outbursts should happen predominantly close to the cluster centre and with high mass ratios between the involved stars.
\end{abstract}

Key words. stars: pre-main sequence - stars: circumstellar matter - stars: early-type

\section{Introduction}

For all stars, with the possible exception of massive stars, accretion of circumstellar matter is the most important formation mechanism. In a previous paper (Pfalzner et al. 2008, hereafter abbreviated as PTS08), it was demonstrated that, in a young, dense cluster environment, interactions with other cluster members lead to accretion bursts where up to $6-7 \%$ of the disc material around the young star can be accreted within a short time $\left(10^{2}-10^{4} \mathrm{yrs}\right)$. The induced accretion rates can be as high as those observed in FU Orionis objects (FUors), raising the question of whether FUors could result from such encounter events.

FUors are a small class of pre-main-sequence objects named after FU Orionis - the first observed object of this type. FUors show large brightness increases (5-6 mag) on short timescales (0.5-15 years) coupled with significant changes in spectral type. Apart from these classical FUors, about 20 FUor-like objects are currently known (Vittone \& Errico 2005), and they have spectral similarities to classical FUors, but for which no eruption has been witnessed (Reipurth et al. 2002).

The mechanism by which these eruptions occur is still poorly understood (Herbig et al. 2003; Reipurth \& Aspin 2004; Hartmann et al. 2004). The most widely accepted view is that the outbursts are caused by some type of accretion-disc instability. In this framework the outburst is not produced by the star itself but results from an increase in the surface brightness of the circumstellar disc surrounding the pre-main sequence stars (Hartmann \& Kenyon 1985; Hartmann \& Kenyon 1996). It should be mentioned that Herbig et al. (2003) suggest an alternative model in which the outburst occurs in an unstable young star rotating near breakup velocity. The various accretiondisc models (Clarke et al. 1990; Bell \& Lin 1994; Kley \& Lin 1999; Lodato \& Clarke 2004; Vorobyov \& Basu 2005; Mejia et al. 2005) offer different mechanisms for triggering the accretion outburst, such as thermal and gravitational instabilities possibly combined with magneto-rotational instabilities (Armitage 2001).

In the following we summarise the properties of the different proposed instability-triggering mechanisms:

(i) The thermal instability model (Bell \& Lin 1994) considers a disc fed at a high mass-accretion rate from a surrounding envelope, which becomes unstable at the inner edge. The instability propagates outwards, producing a slowly rising luminosity compatible with the light curve of V1515 Cyg. However, this model cannot explain the rapid rise-time of FU Ori and V1057 Cyg (Vittone \& Errico 2005). Another problem is the size of the outburst region, where the thermal instability model predicts an outer radius of $\sim 20 R_{\odot}$. Constructing a radiative transfer disc model of FU Orionis, Zhu et al. (2007) find that the outer radius of the accreting 
region is $0.5-1.0 \mathrm{AU}$ and conclude that thermal instability models have difficulties explaining this fact.

(ii) Gravitational instabilities occur only if the disc masses involved are high enough. According to Vorobyov \& Basu (2006), the accretion bursts are associated with the formation of dense protostellar/protoplanetary embryos, which are later driven onto the protostar by the gravitational torques that develop in the disc. There is some ambiguity about the duration of the outburst and the rise times. Armitage et al. (2001) see an outburst duration of $\sim 10^{4} \mathrm{yr}$, two orders of magnitude longer than the observed ones, whereas Vorobyov \& Basu (2006) claim very short (<100 yr) vigorous $\left(\dot{M}=1-10 \times 10^{-4} M_{\odot} / \mathrm{yr}\right)$ accretion bursts, which are intervened by longer periods $\left(>10^{3} \mathrm{yr}\right)$ of quiescent accretion. In their model the frequency of the bursts decreases noticeably with time, and no bursts are seen after $t \sim 0.3$ Myr. The high disc mass required by this model $\left(\approx 1 M_{\odot}\right)$ is consistent with measurements of some FU Orionis objects (Henning et al. 1998; Sandell \& Weintraub 2001), but the measured disc mass of V1057 Cyg $\left(\approx 0.1 M_{\odot}\right.$, Sandell \& Weintraub 2001) seems too low to cause any observable effect.

(iii) A related idea is the passage of a companion star close to the primary star causing an increase in accretion, which was originally proposed by Toomre (Kenyon et al. 2000). This concept was investigated by Bonnell \& Bastian (1992b) for the case of wide eccentric binaries. Clarke \& Syer (1996) studied the situation where a protoplanetary/protostellar companion close to the primary causes a gravitational instability.

Here we investigate the properties of the very similar situation to the latter - encounters in young dense clusters as described in PTS08. The difference in this approach is that, whereas Bonnell \& Bastian (1992) and Clarke \& Syer (1996) look for specific situations that would produce the accretion rates necessary for FUor, we start from the induced accretion processes that inevitably occur in young clusters and ask whether these would be interpreted as FUors.

Before we discuss the likelihood of the passage of a star causing FUor outbursts, we first summarise the known FUors properties.

\section{Properties of FUors and FUor-like stars}

The most striking feature of FUors is, as already mentioned, the increase in brightness of 5-6 mag. While the rise times for outbursts are usually very short $(\sim 1-10 \mathrm{yr})$, the decay timescales range from decades to centuries.

FUors are spatially and kinematically associated with starforming regions (Herbig 1977; Hartmann \& Kenyon 1985). Other observational properties of FUors are their optical spectrum resemblance to F-or G-type supergiant stars (Herbig 1977), their near-infrared spectra being similar to K-or M-type cooler giant stars (Mould et al. 1978), their association with bright reflection nebulae (Goodrich 1987), the heavy extinction, and their mostly large infrared excess emission from circumstellar dust (Weintraub et al. 1991). Some FUors have collimated HerbigHaro $(\mathrm{HH})$ jets and wide-angle molecular outflows. These properties suggest that at least some of the FUors are quite young stellar objects.

Another characteristic is that they show double-peaked absorption-line profiles (Hartmann \& Kenyon 1996). In the most widely accepted model (Kenyon et al. 1993) these peculiarities are explained by a combination of an accretion disc and an envelope. In contrast to the discs of most classical T Tauri stars, which are primarily heated by radiation from the central star (e.g., Kenyon \& Hartmann 1996), the disc heating of FUors is dominated by local accretion energy release at least in the inner disc regions.

Some FUors have high disc masses (Henning et al. 1998; Sandell \& Weintraub 2001), typically a few tenths of a solar mass with a few FUor discs being even more massive. These discs are more massive than those of Class II T Tauri stars, which usually have an order of magnitude lower mass $\left(\sim 0.01 M_{\odot}\right.$ Osterloh \& Beckwith 1995), resembling more the disc masses of Class I objects (Sandell \& Weintraub 2001) - another indication that some FUors likely are extremely youthful compared to $\mathrm{T}$ Tauri stars. However, exceptions also exist with much lower disc masses undergoing FU outburst like V1057 Cyg $\left(\approx 0.1 M_{\odot}\right.$, Sandell \& Weintraub 2001) and FU Orionis itself $\left(<0.02 M_{\odot}\right)$. Both AR 6 and FU Orionis are Class II objects, as they lack strong sub-mm fluxes, indicating that their circumstellar envelopes and discs are relatively depleted. The implication therefore is that FUor eruptions can occur during any phase of the stellar evolution.

In addition, the limited number of measured FUor disc sizes also hints at them being larger than those of $\mathrm{T}$ Tauri stars (Weintraub et al. 2004; Sandell \& Weintraub 2001), more like Class 0 and Class I objects (Chini et al. 1997; Huard et al. 1999; Hogerheijde \& Sandell 2000; Sandell \& Weintraub 2001), or possibly even larger than those (Sandell \& Weintraub 2001). This might suggest that FUor events are more likely among sources surrounded by large discs.

A growing number of FU Orionis objects are found to be binaries (Reipurth \& Aspin 2004) or even part of a multiple system. For example, the FU Orionis object Z CMa is a binary system (Koresko et al. 1991), and a companion to FU Ori has been found (Reipurth \& Aspin 2004; Wang et al. 2004). However, it has not yet been proved that all FU Orionis objects have companions.

Most FUors are found in dense regions of dark clouds and are quite often heavily reddened by local extinction (Sandell \& Weintraub 2001). Few FUors or stars with FUor-like spectra are found in regions of clustered star formation such as Tau-Aur, Oph, NGC 1333, IC 346, or the Orion nebula cluster. Instead, they are mostly found in regions of low star-formation activity. An exception is the double system AR 6A and 6B, which is part of the Spokes young stellar cluster (Moriarty-Schieven et al. 2008).

The common properties of FUors and FUor-like objects are summarised in Table 1. However, the statistics are still poor and these correlations must be confirmed through additional studies; an obvious statement, if one considers the known properties of individual FUor sources listed in Table 2.

\section{Method}

We performed two types of simulations: tree code simulations to study the effect of encounters on star-disc systems (for details see Pfalzner et al. 2005a) and cluster dynamics simulations (see Olczak et al. 2006) performed with the Nbody6++ code (Spurzem 1999) to determine the likelihood and degree of encounter-triggered accretion.

In the cluster simulations, for simplicity all stars were assumed to be initially single. Cluster models were set up with a spherical density distribution $\rho(r) \propto r^{-2}$ and a MaxwellBoltzmann velocity distribution. The stellar masses were 
Table 1. Properties of FUor and FUor-like objects.

\begin{tabular}{ll}
\hline \hline Property & \\
\hline accretion rate & $10^{-7}-10^{-4} M_{\odot} / \mathrm{yr}$ \\
stellar mass & $\sim$ solar-mass or lower \\
rise-times & $1-10 \mathrm{yr}$ \\
decay times & $10-$ several $100 \mathrm{yr}$ \\
spectral type & $\begin{array}{l}\text { large infra-red excess } \\
\text { double peaked } \\
\text { absorption lines } \\
\text { location }\end{array}$ \\
$\begin{array}{l}\text { star-forming regions } \\
\text { rarely in dense clusters } \\
\text { probably younger than T Tauri stars } \\
\text { age }\end{array}$ & $\begin{array}{l}\text { but older systems exist, too } \\
\text { often larger than T Tauri stars } \\
\text { often more massive than T Tauri stars } \\
\text { disc size }\end{array}$ \\
disc mass & high low disc masses exist, too \\
binarity rate &
\end{tabular}

generated randomly according to the mass function given by Kroupa (2002) in a range $50 M_{\odot} \geq M^{*} \geq 0.08 M_{\odot}$. During the simulation, information of all perturbing events on each stellar disc was recorded. The quality of the dynamical models, which we chose to be in virial equilibrium, was determined by comparison to the observational data of the ONC (McCaughrean et al. 2002).

The encounter simulation part of the investigation is mainly based on our earlier work (Pfalzner et al. 2008), which includes a parameter study of the situation where the disc of a star of mass $M_{1}^{*}$ is perturbed by the flyby of a second star of mass $M_{2}^{*}$. In these simulations, we assumed disc parameters typical of T Tauri stars: the discs to be of low-mass $\left(0.01 M_{\odot}\right)$, the disc surface density to have a $1 / r$-dependence initially and the disc size to scale with the stellar mass as $r_{\mathrm{d}}=150 \mathrm{AU} \sqrt{M_{1}^{*}\left[M_{\odot}\right]}$.

The code applied here cannot describe the disc dynamics simultaneously with the accretion process as the mass density and so the required number of simulation particles - would be very high close to the star. More important, the required temporal resolution for the accretion process would be so high that the overall disc dynamics could not be monitored long enough. The main question is whether perturbations to the inner disc area by an encounter can induce accretion. Whether this accretion happens directly by a particle stream or only by some related mechanism is of secondary importance. Therefore we resort to the approach of Bonnell \& Bastien (1992) and Vorobyov \& Basu (2006), who modelled accretion in FU Orionis objects by investigating the amount of matter reaching a sphere of a certain size around the central star - here a region of radius $1 \mathrm{AU}$. So the word "accretion" is used in this sense hereafter. We model only gravitational interactions, while pressure and viscous forces have been neglected. In principle the latter could hinder matter reaching the inner disc areas due to pressure gradients. However, here the velocity of the instreaming matter is so high that this is not a problem in most cases.

\section{Connection between encounter-triggered accretion and FUors?}

In the following we present our simulation results of encountertriggered accretion bursts and compare them with the characteristics of FUors listed in Table 1.

Our simulations have already shown that a fly-by leads to a very rapid increase in accretion. We demonstrated in PTS08 that the maximum amount of matter accreted in a single prograde, coplanar encounter event is up to $6-7 \%$ of the disc mass.

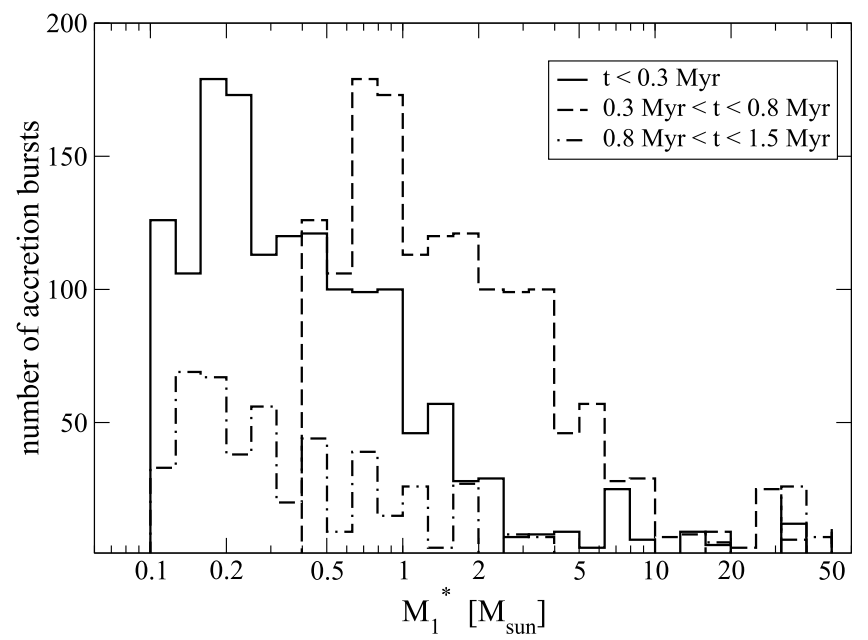

Fig. 1. The number of encounters that lead to accretion bursts as a function of the primary mass $M_{1}^{*}$ for different time intervals of the cluster development.

For typical disc parameters of a solar-mass star T Tauri star $\left(m_{\mathrm{d}} \sim 0.01 M_{\odot}, r_{\mathrm{d}} \sim 150 \mathrm{AU}\right)$, this would be equivalent to an encounter-averaged accretion rate of $10^{-7}-10^{-6} M_{\odot} / \mathrm{yr}$. In the initial phases, the instantaneous accretion rates can be much higher reaching $10^{-4} M_{\odot} / y r$, which is what one observes in FUors. Submillimetre observations show that the disc masses in FUor systems $\left(m_{\mathrm{d}}^{\mathrm{FU}} \sim\right.$ a few times $0.1 M_{\odot}$; Sandell \& Weintraub 2004) are much higher than those of T Tauri stars $\left(m_{\mathrm{d}}^{\mathrm{TT}} \sim 0.01 M_{\odot}\right.$, Osterlohe \& Beckwith 2004). Such massive discs guarantee similar high accretion rates even in encounters that are less favourable for accretion like non-coplanar or more distant encounters, etc.

\subsection{Low-mass stars}

As demonstrated in PTS08, each individual massive star in an ONC-like environment is much more likely to undergo accretion outbursts due to encounters than any lower mass stars. At first sight this seems to contrast to the observed FUors mostly being low-mass stars $\left(\sim 0.1-2 M_{\odot}\right)$. However, as there are many more low-mass stars than massive stars in the cluster, the number of outbursts of all low-mass stars is higher at any given time than that of all massive stars (see Fig. 3 in PTS08). Therefore one is far more likely to observe an accretion burst in a low-mass star than in a massive star, which does agree with the observations in FUors.

\subsection{Rise and decay times}

With shorter rise than decay times, the overall shape of the temporal development of the accretion induced by encounters (see Fig. 2) appears to be similar to what is observed in FUors. Our simulations show that the accretion induced by strong encounters characteristically rises within 10 to several 10s of years, while afterwards the accretion declines typically on timescales of several 10s to several 100s of years.

The accretion rise and decay times depend on the interaction strength, with stronger interactions leading to shorter but more intense accretion outbursts. The reason is that the time where both stars are close together is shorter for stronger interactions. Figure 3 shows the outburst duration (here defined as the time in which $95 \%$ of the total matter is accreted) as a function of the mass ratio $M_{2}^{*} / M_{1}^{*}$ and the periastron distance in the encounter. 
Table 2. Observed properties of FUor and FUor-like objects.

\begin{tabular}{|c|c|c|c|c|c|c|c|}
\hline Object & $t$ (Rise) & $t$ (Decay) & Stellar Mass & Disc Mass ${ }^{2}$ & Accretion rate & Binary & Disc size \\
\hline BBW 76 & & $\sim 40 \mathrm{yr}$ & & $0.15 M_{\odot}$ & & & \\
\hline CB34 V & & & & $0.2 M_{\odot}$ & & & \\
\hline FU Ori & $\sim 1 \mathrm{yr}$ & $\sim 100 \mathrm{yr}$ & $0.35 M_{\odot}$ & $<0.02 M_{\odot}$ & $6.5 \times 10^{-51}$ & $\mathrm{yes}^{1}, \mathrm{k}, 217 \mathrm{AU}^{8}$ & \\
\hline V1057 Cyg & $\sim 1 \mathrm{yr}$ & $\sim 10 \mathrm{yr}$ & & & & & \\
\hline V1515 Cyg ${ }^{3}$ & $\sim 20 \mathrm{yr}$ & $\sim 30 \mathrm{yr}$ & & $0.13 M_{\odot}$ & & & \\
\hline L1551 IRS 5 & & & & $0.23 M_{\odot}$ & & yes, $45 \mathrm{AU}^{7}$ & \\
\hline PP 13S & & & & $0.1 M_{\odot}$ & & & $2000 \mathrm{AU}^{2}$ \\
\hline Re 50 N IRS1 & & & & & & & \\
\hline V1331 Cyg & & & & & & & \\
\hline V1735 Cyg & $<8 \mathrm{yr}$ & $>20 \mathrm{yr}$ & & $0.42 M_{\odot}$ & & & \\
\hline V346 Nor & $<5 \mathrm{yr}$ & $>5 \mathrm{yr}$ & & & & yes? & \\
\hline V883 Ori & & & & $0.39 M_{\odot}$ & & & \\
\hline Z CMa & & $>100 \mathrm{yr}$ & $1.1 M_{\odot}$ & & $7.9 \times 10^{-5}$ & yes,93 $\mathrm{AU}^{9}$ & \\
\hline RNO 1B & & & & & & yes, $5000 \mathrm{AU}^{8}$ & \\
\hline RNO 1C & & & & & & yes, $5000 \mathrm{AU}^{8}$ & \\
\hline AR 6A & & & & & & yes, $2240 \mathrm{AU}^{8}$ & \\
\hline AR 6B & & & & & & yes, $2240 \mathrm{AU}^{8}$ & \\
\hline OO Ser & & & & & & & \\
\hline V1647 Ori & & & & & & & \\
\hline V733 $\mathrm{Cep}^{3}$ & & $>20 \mathrm{yr}$ & & & & & \\
\hline RNO $127^{4}$ & & $>9 \mathrm{yr}$ & & & & & \\
\hline Parsamian $21^{5}$ & & & & $<0.3 M_{\odot}$ & $0.3 M_{\odot}$ & & $360 \mathrm{AU}$ \\
\hline
\end{tabular}

${ }^{1}$ Malbet et al. (2005), ${ }^{2}$ Sandell \& Weintraub (2001), ${ }^{3}$ Reipurth et al. (2007), ${ }^{4}$ Movsessian et al. (2006), ${ }^{5}$ Kospal et al. (2008), ${ }^{6}$ Vittone \& Errico (2005), ${ }^{7}$ Rodriguez (1998), ${ }^{8}$ Quanz et al. (2007), ${ }^{9}$ Koresko (1991).

It can be seen that the mass ratio has to be high in order to obtain strong outbursts of short duration.

Although encounters exist that produce strong short outbursts, we never observe rise times on scales of 1 year in our simulations, as observed for FU Ori; however, this does not necessarily mean that FUor events are not triggered by encounters. The crude definition and modelling of the accretion process in our simulations could cause the accretion times to appear longer than they actually are. As Hartmann \& Kenyon (1996) noticed, the interaction must perturb the disc at $1-10 \mathrm{AU}$ to produce short enough rise times, because perturbations at larger distances naturally show much longer (viscous) rise-times. However, in our simulations the inner edge of our disc is at $10 \mathrm{AU}$, so that we do not resolve the area down to $1 \mathrm{AU}$, as required. Moreover, the pressure and viscous forces neglected here could also alter the rise and decay times.

\subsection{Sparcity in cluster environments}

The strongest argument against FUor outbursts being triggered by encounters is that few FUors or stars with FUor-like spectra are found in young clusters such as Tau-Aur, Oph, NGC 1333, IC 346, or the Orion nebula cluster. If encounters triggered FUor outbursts it is in these dense environments that one would expect most of the bursts. However, FUors are mostly found with regions of low stellar density (Herbig et al. 2003). An exception is the double system AR 6A and 6B, which is part of the Spokes young stellar cluster (Moriarty-Schieven et al. 2008). This implies that either FUors are much less frequent in high-density regions or else they have fewer outbursts.

Greene et al. (2008) suggest that interactions between stars in clusters could disrupt discs, perhaps eliminating much FUor activity during the Class 0 or Class I protostellar evolutionary phases. This would agree with the fact that encountertriggered accretion bursts are a strong function of time in dense environments like the ONC. Figure 4 shows the number of encounter-triggered accretion-bursts in any $10^{4} \mathrm{yr}$ time interval as a function of cluster age for an ONC-like cluster. It can be seen that there are a factor 10-20 fewer accretion outbursts nowadays $(\sim 1 \mathrm{Myr})$ than in the early phases of the cluster development. The reasons are that the cluster becomes less dense and the discs lose mass due to tidal interactions.

\section{Encounter or binary?}

Many of the observed FU Orionis objects are classified as binaries (Koresko et al. 1991; Kenyon et al. 1993; Rodriguez et al. 1998; Hartmann \& Kenyon 1996; Aspin \& Reipurth 2003), and some of the companions are surrounded by a disc themselves (Reipurth \& Aspin 2004; Quanz et al. 2006). The distances of the companions are relatively large ( 40-5000 AU), so they are all visual binaries. Since usually at least one of the stellar masses is unknown, the two stars could in principle actually undergo an encounter rather than being a binary. What speaks against this is again the low stellar density in most of these regions where FUors are observed.

However, the accretion burst induced by an encounter and by a binary that recently formed would be nearly indistinguishable anyway, as the binary would have to have a high eccentricty to display the accretion characteristics of an FUor. The binary mechanism is attractive, since most stars are members of multiple systems.

The interaction must strongly perturb the disc at 1-10 AU to produce the short rise times of FUors. Often this requirement is translated as the necessity of small periastron distances in the binary. Our results suggest that high mass ratios could equally lead to the necessary perturbation, so that the requirements on the orbital distribution are less stringent (see Fig. 3). 

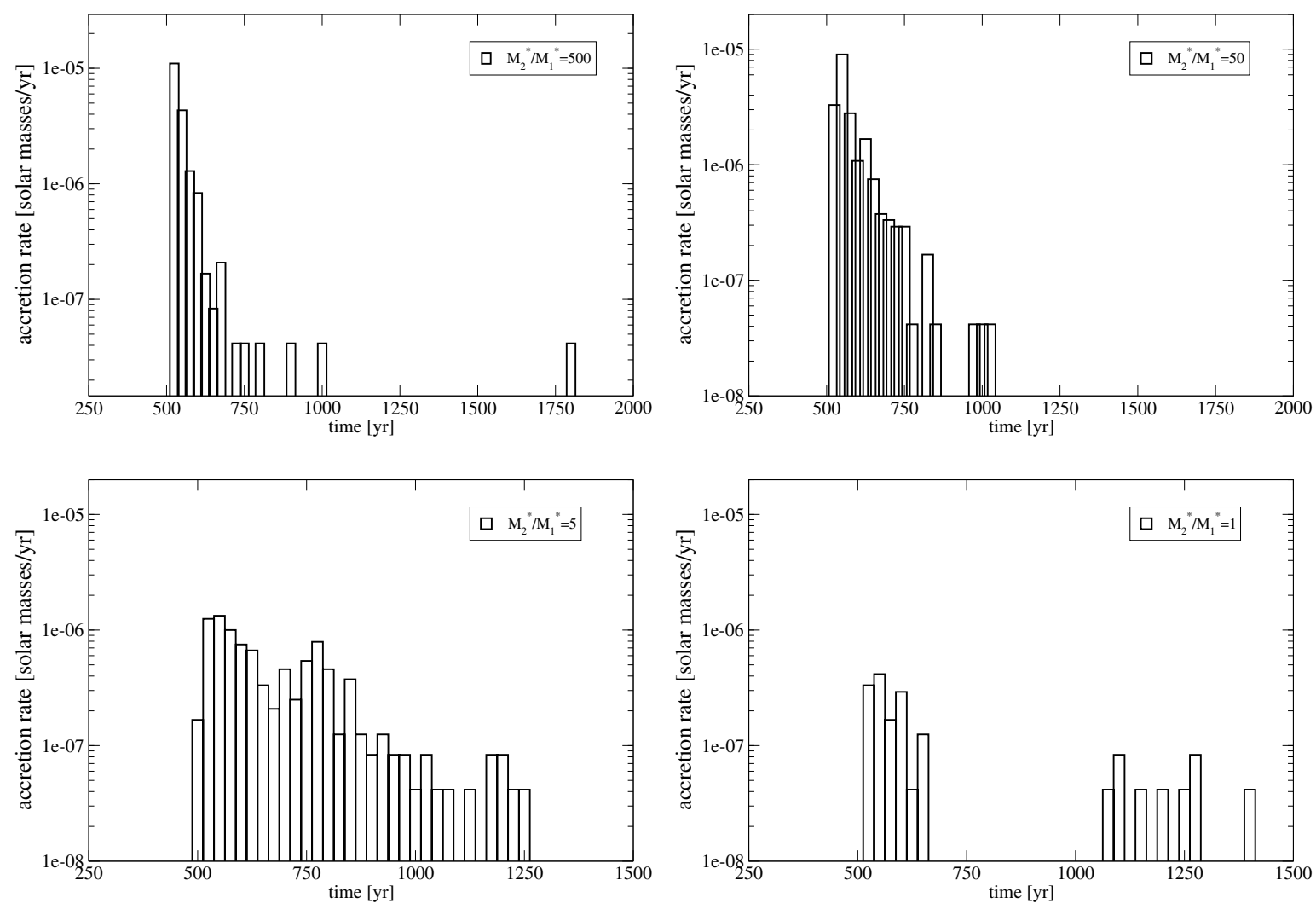

Fig. 2. Accretion rate as a function of time for a parabolic encounter with $M_{2}^{*} / M_{1}^{*}=500,50,5,1$ and a perihel distance of $1.5 r_{\mathrm{d}}$ assuming $m_{\mathrm{d}}=0.001 M_{\odot}$.

\subsection{Mass from primary or secondary}

Bonnell \& Bastien (1992a) studied how wide eccentric binaries could trigger FUor outbursts. As the parameter space is rather extensive, they studied only a few selected configurations. They find that for unequal-mass binaries the more massive component has a stronger tendency to be obscured by the surrounding medium, has the more massive disc, induces a higher accretion rate, and that accretion will be much greater onto the lower mass secondary than onto the primary. They suggest that it is actually this sudden increase in matter in the secondary's disc obtained from the primary's disc that could describe the FU phenomenon. Because the accreted matter obtained from the primary's disc has little rotational velocity in the secondary's reference frame, it can be deposited at arbitarily small radii or accreted directly. Mass would fall predominantly onto the centre of mass close to the primary and would form a disc that obscures the primary. By contrast, the secondary would accrete directly with less extinction, so that the strong far-infrared excess could be explained by the presence of an IR companion.

Reipurth \& Aspin (2004) propose that such events may be a natural consequence of interactions between two components that spiral in toward each other following the breakup of an unstable triple system. Alternatively, Greene et al. (2008) suggest that a dense cluster environment might accelerate the orbital evolution, again causing FUor eruptions in clusters to occur mostly during the Class 0 or Class I stages (Reipurth \& Aspin 2004).

Although the approach of an accretion burst in the secondary is appealing, there are some problems. First, accretion of matter onto the secondary can only happen for relatively close encounters, $r_{\text {peri }} / r_{\mathrm{d}}<2$ (see Fig. 5). However, some of the discs observed around FUors are relatively large, so this might be not so stringent. Second, the overall accretion time of the matter onto the secondary is longer than onto the primary (see Fig. 6) and, more important, the rise time of the accretion burst is longer, too. Only if $M_{2}^{*} / M_{1}^{*} \gg 1$, can the rise time become $\sim 10 \mathrm{yrs}$, but in this case the accretion rate onto the secondary declines considerably. As the rise time is the most critical point anyway, there is a problem here.

However, the two sources of accretion - onto the primary and onto the secondary - could potentially hold the key to distinguishing between binary/encounter driven outbursts and instabilities caused by gravitational unstable discs or planetary companions. The first do have these two sources of accretion, whereas the last do not. Our simulations show that relatively independently of the actual encounter parameters, the accretion onto the secondary always happens $\sim 100$ yrs before that onto the primary. This possibly means that 100 yrs before the outburst in the visual an outburst takes place in the infrared. If this were to be observed, it would give a clear distinction between the two groups of triggering mechanisms.

\section{Conclusion}

We have shown that there are many properties that encounterinduced accretion bursts and FUors events have in common. The accretion rates, the accretion profile and the overall burst duration in our encounter simulations are basically identical to those observed in FUors. From our simulations, it follows that encounter-induced accretion outburst would mainly be visible for low-mass stars and would currently not be distinguishable from a visual binary. Both properties are in accordance with many FUors being low-mass binaries. However, there are two problems with FUors being induced by encounters: (i) FUors are predominantly (but not exclusively) observed in regions of low 
a)

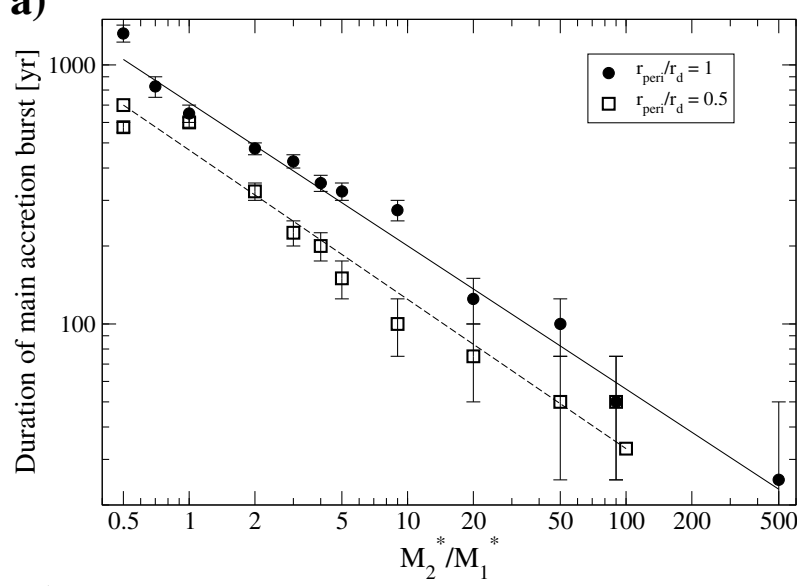

b)

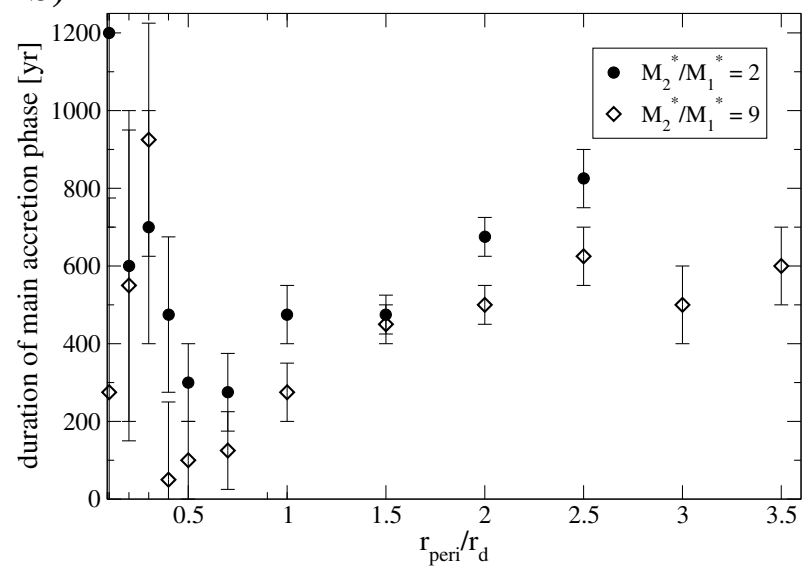

Fig. 3. Time required until the star has accreted $95 \%$ of the mass, that it eventually accretes from its own disc as a function of a) the mass ratio $M_{2}^{*} / M_{1}^{*}$ and b) the relative periastron $r_{\text {peri }} / r_{\mathrm{d}}$ in a single encounter. The lines serve to guide the eye.

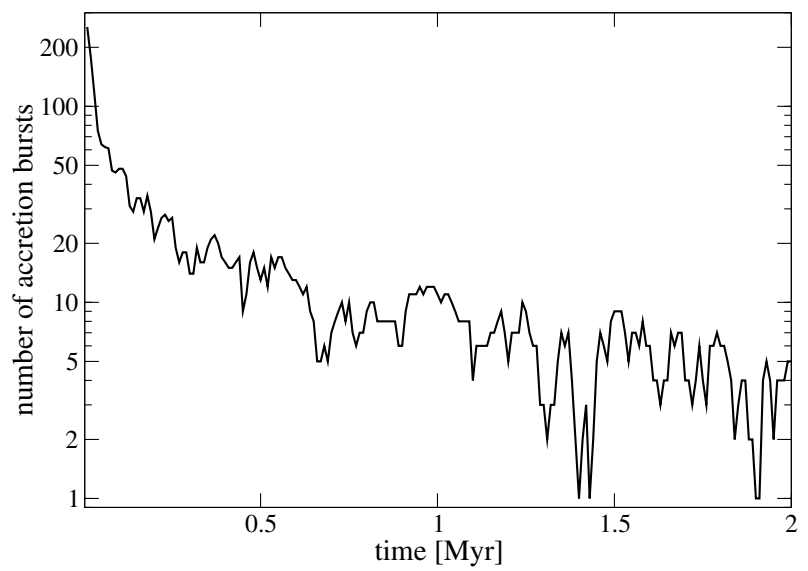

Fig. 4. The number of encounters in any $10^{4} \mathrm{yr}$ long time-interval that lead to accretion bursts as a function of the cluster age in an ONC-like cluster.

stellar density and (ii) the rise-times in encounter-induced accretion bursts seem to be longer than those observed for FUors.

If the latter problem is real, then because of the similarity in mechanism other models of gravitationally-triggered accretion bursts possibly share this problem of too long rise times, no matter whether the trigger is an encounter, a binary, a massive disc or a planetary companion. However, this does not necessarily mean a)

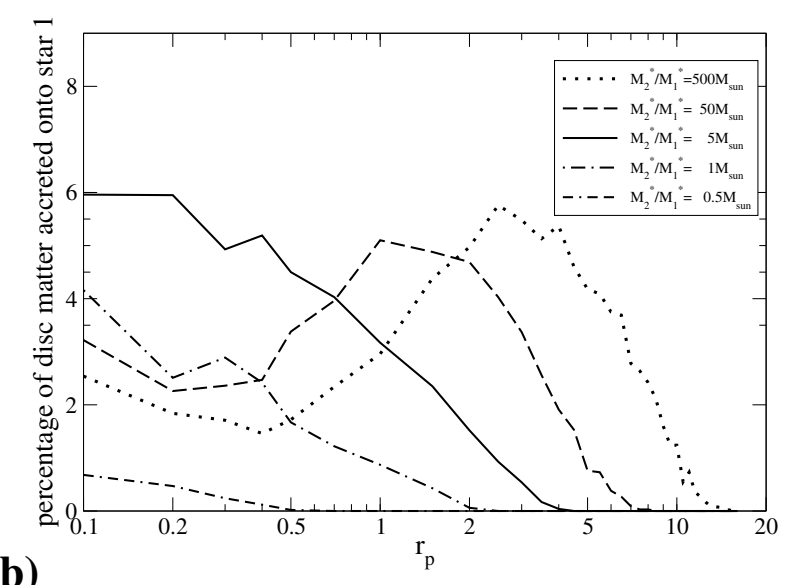

b)

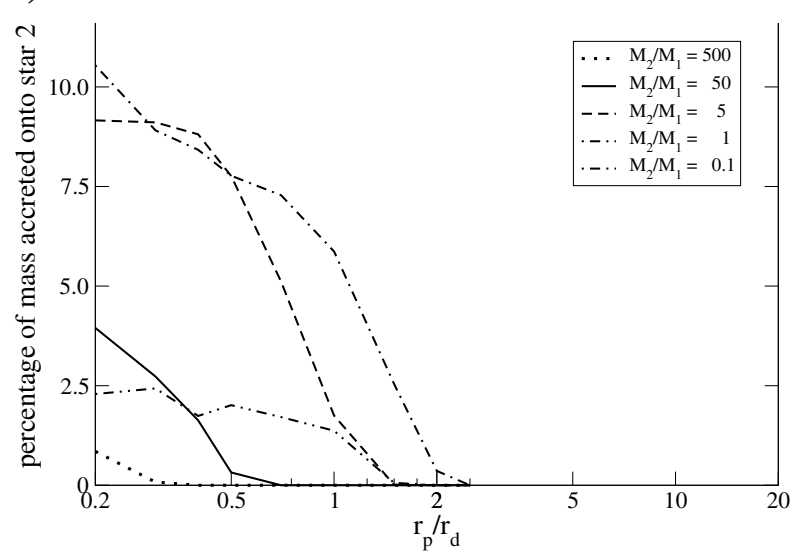

Fig. 5. Percentage of disc mass accreted onto star 2 as a) a function of the relative periastron distance $r_{\mathrm{p}} / r_{\mathrm{d}}$ and $\mathbf{b}$ ) a function of relative mass ratio $M_{2}^{*} / M_{1}^{*}$ in parabolic, prograde coplanar encounters.

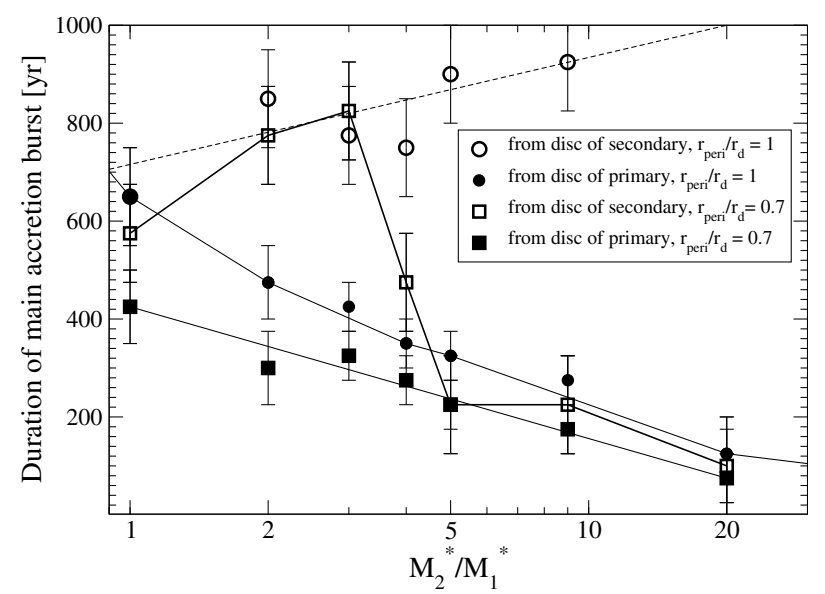

Fig. 6. Comparison between the time required until the star has accreted $95 \%$ of the mass. It eventually accretes from its own disc (full circles), and the mass it accretes from the disc of the secondary star as a function of the mass ratio $M_{2}^{*} / M_{1}^{*}$.

that none of these models can lead to FUor events; on the contrary, each of them could trigger FUor events and the long rise times might actually be an artefact of the crude accretion modelling (disc cut-off at $10 \mathrm{AU}$ and exclusion of pressure/viscous forces).

The obvious question that arises is why the spectra of FUors and FUor-like stars are not more similar to the spectra of Class I 
protostars with large veiling and relatively high accretion rates ( $\left.>10^{-6} M_{\odot} / y r\right)$. Greene et al. (2008) suggest that higher accretion rates are possibly needed to produce luminous discs with absorption features similar to those found in FUors.

Given that the properties of FUors can differ considerably from young stars with massive large discs in sparse regions to stars with low disc masses in cluster environments - it may be too simplistic to assume there is only one way to trigger FUor outbursts. There may be actually several ways for FUors to occur - some externally triggered by encounters, binaries, disintegration of a triple system, and planetary companions but also others caused by diverse instabilities in discs around single stars. FUor observations could then be just a selection effect requiring a certain accretion rate to see FUor spectra. This would also explain the preference but not exclusiveness for FUor phenomena to occcur in young stars with high disc mass and large disc size.

This interpretation only works as long as there are no FUors found with an accretion rate less than $10^{-6} M_{\odot} /$ yr or stars with accretion rates higher than $10^{-5}$ without FUor characteristics. If this were the case, then we would expect many FUors in dense clusters younger than 0.3 Myrs, because here encountertriggered accretion bursts should be quite common. They would mainly occur close to the cluster centre and if the mass ratio of the encounter partners is high. If the encounter were close enough, an outburst in the infrared would precede one in the visible.

Acknowledgements. We want to thank B. Reipurth for his very helpful comments. Simulations were partly performed at the John von Neumann Institute for Computing, Research Centre Jülich, Project HKU14.

\section{References}

Armitage, P. J. 2001, MNRAS, 324, 705

Aspin, C., \& Reipurth, B. 2003, AJ, 126, 2936

Bell, K. R., \& Lin, D. N. C. 1994, ApJ, 427, 987

Bonnell, I., \& Bastien, P. 1992a, ApJ, 401, L31

Bonnell, I., \& Bastien, P. 1992b, ApJ, 401, 654

Chini, R., Reipurth, B., Sievers, A., et al. 1997, A\&A, 325, 542

Clarke, C. J., \& Syer, D. 1996, MNRAS, 278, L23

Clarke, C. J., Lin, D. N. C., \& Pringle, J. E. 1990, MNRAS, 242, 439
Goodrich, R. W. 1987, PASP99, 116

Greene, T. P., Aspin, C., \& Reipurth, B. 2008, AJ, 135, 1421

Hartmann, L., \& Kenyon, S. J. 1985, ApJ, 299, 462

Hartmann, L., \& Kenyon, S. J. 1996, ARA\&A, 34, 207

Hartmann, L., Hinkle, K., \& Calvet, N. 2004, ApJ, 609, 906

Henning, Th., Burkert, A., Launhardt, R., Leinert, Ch., \& Stecklum, B. 1998, A\&A, 336, 565

Herbig, G. H. 1977, ApJ, 217, 693

Herbig, G. H., \& Jones, B. F. 1983, AJ, 88, 1040

Herbig, G. H., Petrov, P. P, \& Duemmler, R. 2003, ApJ, 595, 384

Hogerheijde, M. R., \& Sandell, G. 2000, ApJ, 534, 880

Huard, T. L., Sandell, G., \& Weintraub, D. A. 1999, ApJ, 526, 833

Kenyon, S. J., Hartmann, L., Gomez, M., Carr, J. S., \& Tokunaga, A. 1993, AJ, 105,1505

Kenyon, S. J., Kolotilov, E., Ibragimov, M. A., \& Mattei, J. A. 2000, ApJ, 531, 1028

Kley, W., \& Lin, D. N. C. 1999, ApJ, 518, 833

Koresko, C. D., Beckwith, S., Ghez, A., Matthews, K., \& Neugebauer, G. 1991, AJ, 102, 2073

Kospal, A., Abraham, P., Apai, D., et al. 2008, MNRAS, 383, 1015

Kroupa, P. 2002, 295, 82

Lodato, G., \& Clarke, C. J. 2004, MNRAS, 353, 841

Malbet, F., Lachaume, R., Berger, J.-P., et al. 2005, A\&A, 437, 627

McCaughrean, M., Zinnecker, H., Andersen, M., Meeus, G., \& Lodieu, N. 2002, The Messenger, 109, 28

Mejia, A. C., Durisen, R. H., Pickett, M. K., \& Cai, K. 2005, ApJ, 619, 1098

Moriaty-Schieven, G. H., Aspin, C., \& Davis, G. R. 2008,

[arXiv:0807.5133]

Mould, J. R., Hall, D. N. B., Ridgway, S. T., Hintzen, P., \& Aaronson, M. 1978, ApJ, 222, L123

Movsessian, T. A., Khanzadyan, T., Aspin, C., et al. 2006, A\&A, 455, 1001

Olczak, C., Pfalzner, S., \& Spurzem, R. 2006, ApJ, 642, 1140

Osterloh, M., \& Beckwith, S. V. W. 1995, ApJ, 439, 288

Pfalzner, S., Vogel, P., Scharwächter, J., \& Olczak, C. 2005, A\&A, 437, 976

Pfalzner, S., Tackenberg, J., \& Steinhausen, M. 2008, A\&A, 487, L45

Quanz, S. P., Henning, T., Bouwman, J., et al. 2007, ApJ, 668, 359

Reipurth, B., \& Aspin, C. 2004, ApJ, 608, L65

Reipurth, B., Hartmann, L., Kenyon, S. J., Smette, A., \& Bouchet, P. 2002, AJ, 124, 2194

Reipurt, B., Aspin, C., Beck, T., et al. 2007, AJ, 133, 1000

Rodriguez, L. F., Porras, A., Claussen, M. J., et al. 1998, Nature, 395, 355

Sandell, G., \& Knee B. G. 2001, ApJ, 546, L49

Sandell, G., \& Weintraub, D. 2001, ApJS, 134, 115

Spurzem, R. 1999, Comp. Astroph., 109, 407

Vittore, A. A., \& Errico, L. 2005, Mem. Soc. Astron. It., 76, 320

Vorobyov, E. I., \& Basu, S. 2005, ApJ, 633, L137

Vorobyov, E. I., \& Basu, S. 2006, ApJ, 650, 956

Wang, H., Apai, D., Henning, T., \& Pascucci, I. 2004, A\&A, 601, L83

Weintraub, D. A., Sandell, G., \& Duncan, D. 1991, ApJ, 382, 270

Zhu, Z., Hartmann, L., Calvet, N., et al. 2007, ApJ, 669, 483 